\title{
Fluorine-free, liquid-repellent surfaces made from ionic liquid- infused nanostructured silicon
}

\author{
Roland W. Bittner ${ }^{1} \cdot$ Katharina Bica $^{1} \cdot$ Helmuth Hoffmann ${ }^{1}[$
}

Received: 25 October 2016/Accepted: 29 November 2016/Published online: 18 December 2016

(C) The Author(s) 2016. This article is published with open access at Springerlink.com

\begin{abstract}
Liquid-repellent surfaces based on slippery liquid-infused porous substrates (SLIPS) were prepared from porous, nanostructured silicon surfaces with different surface functionalization, infused with the polar ionic liquid 1-ethyl-3-methylimidazolium methylsulfate $\left(\left[\mathrm{C}_{2}\right.\right.$ $\operatorname{mim}] \mathrm{MeSO}_{4}$ ). Contrary to nonpolar SLIPS based on perfluorinated substrates and infusion liquids, $\left[\mathrm{C}_{2}\right.$ $\operatorname{mim}] \mathrm{MeSO}_{4}$ forms stable SLIPS with high energy surfaces like native silicon $\left(\mathrm{Si}-\mathrm{SiO}_{2}\right)$ or ionic liquid-functionalized silicon $\left(\mathrm{Si}-\left[\mathrm{C}_{3} \mathrm{mim}\right] \mathrm{Cl}\right)$, whose liquid-repellent properties against low surface tension liquids (toluene, cyclohexane) were demonstrated by very low sliding angles $\left(\alpha<3^{\circ}\right)$ and low contact angle hysteresis $\left(\Delta \theta<10^{\circ}\right)$. These polar, ionic liquid-based SLIPS present a promising, environmentally benign extension of liquid-infused substrates to natural, high-energy oxide surfaces.
\end{abstract}

Graphical abstract

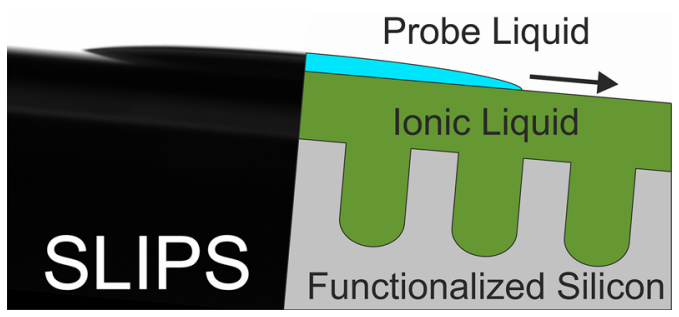

Electronic supplementary material The online version of this article (doi:10.1007/s00706-016-1888-2) contains supplementary material, which is available to authorized users.

Helmuth Hoffmann

helmuth.hoffmann@tuwien.ac.at

1 Institute of Applied Synthetic Chemistry, Vienna University of Technology, Vienna, Austria
Keywords Surface - Monolayers - Nanochemistry · Ionic liquids · Wetting

\section{Introduction}

The wetting properties of a solid surface are commonly characterized by the static contact angle or Young angle $\theta_{\mathrm{Y}}$ [1] of a drop of a probe liquid deposited on the surface and the contact angle hysteresis $\Delta \theta[2,3]$, which is the difference between the advancing contact angle $\theta_{\mathrm{a}}$ and the receding contact angle $\theta_{\mathrm{r}}$ of a drop rolling down the tilted surface. Liquid repellency is usually expressed by a large Young angle $\theta_{\mathrm{Y}}$ and a small hysteresis $\Delta \theta$, because a large $\theta_{\mathrm{Y}}$ means a small solid/liquid contact area and a small $\Delta \theta$ means a small surface tilt angle that is required to shed the liquid (the so-called roll-off angle $\alpha$ ). One of the most challenging tasks in the fabrication of synthetic surfaces with specific wetting properties is to make a surface repellent to all kinds of liquids and natural fluids, in particular inert, nonpolar liquids with low surface tensions [4-7]. Over the past decade, the most common approach to prepare liquid-repellent surfaces has been based on the Cassie-Baxter wetting state [8] (Fig. 1a): a liquid in contact with a rough solid substrate, which is usually coated with an inert, low surface energy substance (most often, a long-chain hydrocarbon or perfluorinated hydrocarbon compound), does not fill the pores of the substrate, but is suspended on the outer, composite surface consisting of a solid fraction $\phi$ and an air fraction $(1-\phi)$. The resulting contact angle $\theta_{\mathrm{CB}}$ is then a weighted average between the Young contact angle of the plane solid $\theta_{\mathrm{Y}}$ and $180^{\circ}$ (contact angle in air). The goal of the Cassie-Baxter approach is to make $\theta_{\mathrm{CB}}$ as large as possible (ideally $180^{\circ}$ ) and the 
contact angle hysteresis $\Delta \theta$ as small as possible using substrates with large $\theta_{\mathrm{Y}}$ (low surface energy coatings) and small $\phi$ (e.g., nanostructured or hierarchically structured surfaces) [9]. Thus, a combination of a chemical factor $\theta_{\mathrm{Y}}$ and a structural factor $\phi$ determines the overall wetting and liquid-repellent properties in the Cassie-Baxter case. The main limitation arises from the fact that the $\mathrm{CB}$ state is thermodynamically stable only for liquid/solid systems for which the Young angle $\theta_{\mathrm{Y}}$ is larger than a certain critical angle $\theta_{\mathrm{C}}=\cos ^{-1}((\phi-1) /(R-\phi))$, where $R$ is the surface roughness (true surface area per unit projected surface area) [10]. This condition can be fulfilled only by a few, selected systems (high surface tension liquids (e.g., water) on low energy surfaces like hydrocarbons or fluoropolymers), whereas for most organic liquids, $\theta_{\mathrm{Y}}$ is always $<90^{\circ}$ on any plane solid surface and a stable Cassie-Baxter state can never be reached. Instead, the liquid penetrates into the substrate pores and forms the Wenzel wetting state [11] (Fig. 1b), which is characterized by high adhesion between liquid and solid and a large contact angle hysteresis $\Delta \theta$, i.e., a liquid-attractive instead of a liquid-repellent wetting state. To overcome this limitation and make surfaces repellent even for nonpolar, organic liquids with low surface tension, substantial effort has been put into the development of synthetic substrates with designed surface textures, which prevent the penetration of liquid into the substrate pores and maintain a metastable Cassie-Baxter state $[4,10]$. Despite numerous successful examples [12-17], the production of such substrates is generally complex and expensive and practical applications are limited by the instability of this metastable state against external pressure, impact, mechanical damage, high temperatures, etc.

A few years ago, a fundamentally different biomimetic approach for liquid-repellent surfaces was first reported, inspired by the prey-capturing method of the carnivorous Nepenthes pitcher plant [18]. This plant captures insects using a slippery, water-lubricated surface at the rim of its pitcher. The rim consists of a porous, irregular microstructure, which fills with water on humid days and remains dry on dry days. Whereas the dry surface is adhesive and non-slippery, the water-filled rim exposes a thin, continuous film of liquid which is held in place by capillary forces of the rim's microstructure and repels the

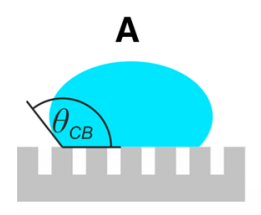

Cassie-Baxter

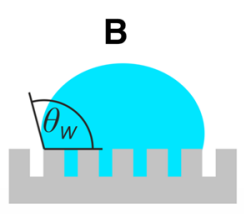

Wenzel

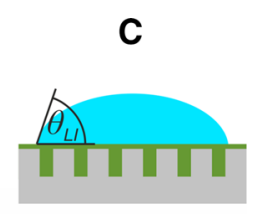

Liquid-Infused
Fig. 1 Wetting models for rough, solid surfaces oil-covered insect feet. Figure 1c shows a model of this liquid-infused wetting state, which is also denoted as slippery liquid-infused porous substrate (SLIPS): the surface of a rough, solid substrate is filled with a lubricating liquid such that the substrate is completely immersed in the liquid. The surface exposed to the ambient phase is no longer a solid, but a thin liquid film of typically a few micrometers thickness. Such a substrate repels liquids, which are immiscible with the lubricating liquid, in a fundamentally different way than a Cassie-Baxter substrate: the solid substrate is no longer in contact with the probe liquid. Its only task is to stabilize the lubricating liquid film through capillary forces and prevent drainage of the film through gravity, shear forces, impact of probe liquid and other external forces. The probe liquid then interacts just with a liquid surface and forms a liquid/liquid interface. Thus, solid/liquid wetting in all Cassie-Baxter based systems is transformed to liquid/liquid wetting in SLIPS-based systems and is governed by completely different rules: The static contact angle and the contact area of a liquid drop on a liquid surface are usually much larger than on a Cassie-Baxter substrate, but the contact angle hysteresis is very small because of the absence of a solidliquid contact line and related pinning effects, which are the primary cause for contact angle hysteresis [2, 3]. The main requirements for a stable and versatile SLIPS systems are (i) a chemically inert lubricating liquid with low volatility and immiscibility with as many other liquids as possible and (ii) a solid substrate with a suitable microstructure and chemical composition to keep the lubricating film firmly in place. The large majority of practically tested SLIPS consist of fluorinated substrates filled with nonpolar, fluorinated hydrocarbons or polyethers because of their low volatility and low miscibility with other liquids [18-31]. This group of nonpolar SLIPS has been shown to repel a large variety of polar and nonpolar liquids as well as complex fluids like blood, crude oil or vegetable oils $[18,19]$, can withstand high pressures and temperatures $[18,20]$ and can even self-heal from minor damage $[18,21]$. Successful practical applications include the prevention of biofilm adhesion and biofouling [22-26], self-cleaning surfaces [27, 28], oil-water separation [24], and the fabrication of anti-icing surfaces [29-31]. Yet, some inherent drawbacks of these nonpolar systems are (i) the exclusive use of fluorinated compounds both as substrate coatings and as lubricating liquids, (ii) leaching of the lubricating liquid caused by its low surface tension, and (iii) the fact that most natural surfaces are polar and cannot be used for nonpolar SLIPS without surface pretreatment.

In the present study, we explore the properties of polar SLIPS, consisting of a polar substrate surface with high surface energy and a nonvolatile, polar lubricating liquid. As the substrate material, we have chosen native silicon, 
which in ambient atmosphere is typically covered by a 1-2 nm-thick oxide layer and therefore represents the ubiquitous group of natural silica-based surfaces. Silica surfaces can also be readily functionalized with a variety of organosilane compounds and allow fine-tuning of their surface energy. Moreover, silicon is also the best-studied material for the fabrication of structured surfaces, and a plethora of methods is available today to make rough, porous substrates to be used as support of the lubricating liquid film in SLIPS. We have chosen for this study a wetchemical, silver-catalyzed etching technique [32-34], which produces a nanostructured surface consisting of densely packed, uniformly oriented silicon nanowires of about 50-200 nm diameter [34]. In search of environmentally friendlier, fluorine-free and polar lubricating liquids, ionic liquids seemed the most promising candidates, as they combine negligible vapor pressure with tunable physicochemical properties. Of particular importance for the present study is the surface tension, as it determines the wetting properties of the lubricating liquid toward both the substrate and the probe liquid. Surface tensions of ionic liquids range typically between 23 and $60 \mathrm{mN} \mathrm{m}^{-1}[35,36]$ and can be readily adapted for optimized wetting properties of a particular liquid-infused substrate.

\section{Results and discussion}

\section{Wetting model for liquid-infused substrates}

A model originally proposed in Ref. [37] is used in this work to describe the different wetting states of a liquidinfused substrate in contact with a second probe liquid (Fig. 2). A rough solid substrate $S$ with a surface roughness $R$ (ratio of the total surface area and the projected surface area), a solid surface fraction $\phi$ (fraction of solid per unit projected surface area), and a surface energy of $\gamma_{S}$ is infiltrated with a liquid A (surface energy $\gamma_{\mathrm{A}}$ ) and is exposed to a probe liquid B (surface energy $\gamma_{\mathrm{B}}$ ) immiscible with $\mathrm{A}$. The interface energies $\mathrm{S} / \mathrm{A}, \mathrm{S} / \mathrm{B}$, and $\mathrm{A} / \mathrm{B}$ are denoted as $\gamma_{\mathrm{SA}}, \gamma_{\mathrm{SB}}$, and $\gamma_{\mathrm{AB}}$. Ideally, as shown in the top model in Fig. 2, liquid A completely fills the pores of the substrate and also completely covers the top solid surface fraction $\phi$, and liquid B leaves this liquid-infused configuration undisturbed and forms a drop with a certain contact angle on the surface of liquid $\mathrm{A}$. We will discuss in the following which conditions must be met to achieve this desired configuration and which other, unwanted wetting states can occur. For this purpose, we pick three representative regions in our model, denoted 1-3 in Fig. 2, and first calculate the total interface energies for all relevant wetting configurations in each of these regions. Region 1 is

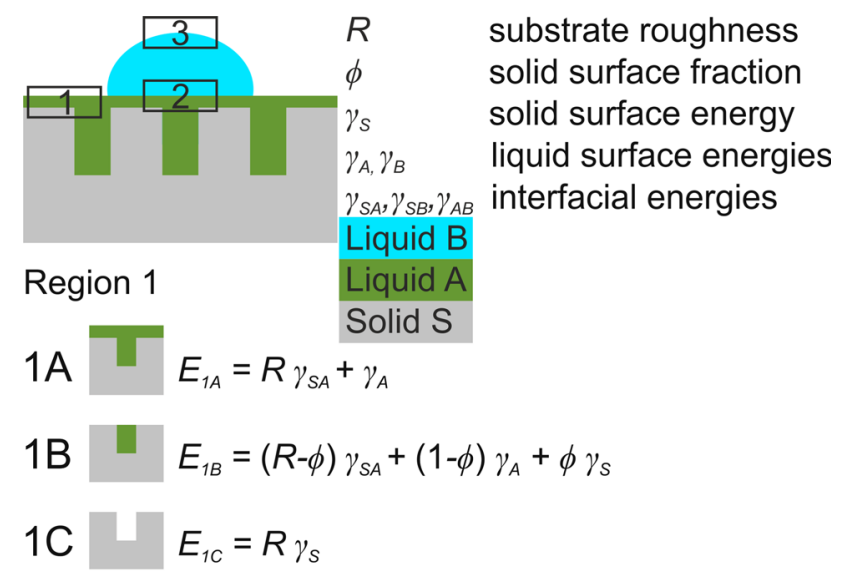

Region 2

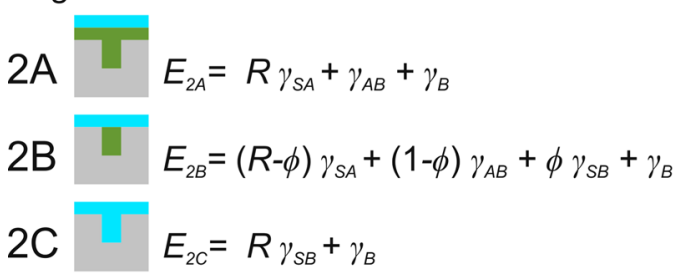

Region 3

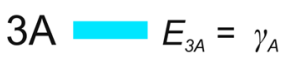

3B $E_{3 B}=\gamma_{A B}+\gamma_{B}$

Fig. 2 Interface energies $E_{(1-3)(\mathrm{A}-\mathrm{C})}$ per unit area for different wetting states $(A-C)$ in different regions (1-3) of a structured solid substrate $S$ infused by a liquid $A$ and wetted by an immiscible liquid $B$

the three-phase range solid/liquid A/air that does not get in contact with liquid $\mathrm{B}$. The three relevant wetting states are complete wetting of the outer surface $\phi$ and the inner surface $R-\phi(1 \mathrm{~A})$, a wetted inner surface and a dry outer surface (1B), and the completely dry substrate (1C). The corresponding interface energies per unit area for $1 \mathrm{~A}-1 \mathrm{C}$ are listed in Fig. 2. Region 2 is located where the substrate infused with liquid $\mathrm{A}$ is covered by the second liquid $\mathrm{B}$. The three relevant wetting states $2 \mathrm{~A}-2 \mathrm{C}$ for this region are obtained simply by substituting air as the ambient medium in region 2 with liquid $\mathrm{B}$. The corresponding expressions for the interface energies of states $2 \mathrm{~A}-2 \mathrm{C}$ (Fig. 2) are therefore simply related to the energies of $1 \mathrm{~A}-1 \mathrm{C}$ by the substitutions $\gamma_{\mathrm{B}}=0, \gamma_{\mathrm{AB}}=\gamma_{\mathrm{A}}$, and $\gamma_{\mathrm{SB}}=\gamma_{\mathrm{S}}$. Region 3 represents the outermost liquid/air interface of liquid $\mathrm{B}$ and can exist in one of two states: either as a liquid B/air interface (state $3 \mathrm{~A}$ ) or as liquid $\mathrm{B} /$ /iquid $\mathrm{A} /$ air interface (state 3B). Thus, the corresponding expressions for the interface energies in region 3 contain only liquid surface energies and are independent of the substrate.

To find the conditions under which the different wetting states are thermodynamically stable, their interface energies are compared and the minimum energy states are 
determined as a function of some experimentally accessible parameters. For region 1, this gives the following set of three inequalities, which can be transformed into simple expressions for $\cos \theta_{\mathrm{A}}=\frac{\gamma_{\mathrm{S}}-\gamma_{\mathrm{SA}}}{\gamma_{\mathrm{A}}}$, where $\theta_{\mathrm{A}}$ is the contact angle of liquid $\mathrm{A}$ on the flat solid substrate $\mathrm{S}$ :

$E_{1 \mathrm{~B}}<E_{1 \mathrm{~A}} \Leftrightarrow \frac{\gamma_{\mathrm{S}}-\gamma_{\mathrm{SA}}}{\gamma_{\mathrm{A}}}<1$,

$E_{1 \mathrm{C}}<E_{1 \mathrm{~A}} \Leftrightarrow \frac{\gamma_{\mathrm{S}}-\gamma_{\mathrm{SA}}}{\gamma_{\mathrm{A}}}<\frac{1}{R}$,

$E_{1 \mathrm{C}}<E_{1 \mathrm{~B}} \Leftrightarrow \frac{\gamma_{\mathrm{S}}-\gamma_{\mathrm{SA}}}{\gamma_{\mathrm{A}}}<\frac{(1-\Phi)}{(R-\Phi)}$.

Exactly the same treatment of the interface energies for region 2 gives:

$E_{2 \mathrm{~B}}<E_{2 \mathrm{~A}} \Leftrightarrow \frac{\gamma_{\mathrm{SB}}-\gamma_{\mathrm{SA}}}{\gamma_{\mathrm{AB}}}<1$,

$E_{2 \mathrm{C}}<E_{2 \mathrm{~A}} \Leftrightarrow \frac{\gamma_{\mathrm{SB}}-\gamma_{\mathrm{SA}}}{\gamma_{\mathrm{AB}}}<\frac{1}{R}$,

$E_{2 \mathrm{C}}<E_{2 \mathrm{~B}} \Leftrightarrow \frac{\gamma_{\mathrm{SB}}-\gamma_{\mathrm{SA}}}{\gamma_{\mathrm{AB}}}<\frac{(1-\Phi)}{(R-\Phi)}$.

The independent parameter for the relative interface energies is now $\frac{\gamma_{\mathrm{SB}}-\gamma_{\mathrm{SA}}}{\gamma_{\mathrm{AB}}}=\cos \theta_{\mathrm{A}(\mathrm{B})}$, where $\theta_{\mathrm{A}(\mathrm{B})}$ is the contact angle of liquid $\mathrm{A}$ on the flat solid substrate immersed in liquid B as the ambient medium. Somewhat simpler is the corresponding analysis of region 3 :

$E_{3 \mathrm{~A}}<E_{3 \mathrm{~B}} \Leftrightarrow \frac{\gamma_{\mathrm{B}}-\gamma_{\mathrm{A}}}{\gamma_{\mathrm{AB}}}<1$.

The relative interface energies can now be plotted as a function of $\cos \theta_{\mathrm{A}}$ for region 1 , as a function of $\cos \theta_{\mathrm{A}(\mathrm{B})}$ for region 2, and as a function of $\frac{\gamma_{\mathrm{B}}-\gamma_{\mathrm{A}}}{\gamma_{\mathrm{B}}}=\Gamma_{\mathrm{AB}}$ for region 3 to give energy diagrams of the relative stabilities of the different wetting states (Supplementary Material, Fig. S1). A simplified version of this energy level diagrams is shown in Fig. 3, where only the lowest energy states of Fig. S1 are considered. Thus, the wetting behavior of liquids on a liquid-infused substrate can be described by three easily accessible experimental parameters $\cos \theta_{\mathrm{A}}, \cos \theta_{\mathrm{A}(\mathrm{B})}$, and $\Gamma_{\mathrm{AB}}$.

\section{Wetting state classifications}

A liquid-infused substrate to be used as a liquid-repellent surface should fulfill the following requirements:

(a) The lubricating liquid $\mathrm{A}$ should completely fill the pores of the substrate and also cover the outer substrate surface (wetting state 1A in Fig. 3). The outer substrate surface is then a pure liquid with small contact angle hysteresis and without any solid protrusions where drops of the probe liquid B might get stuck.
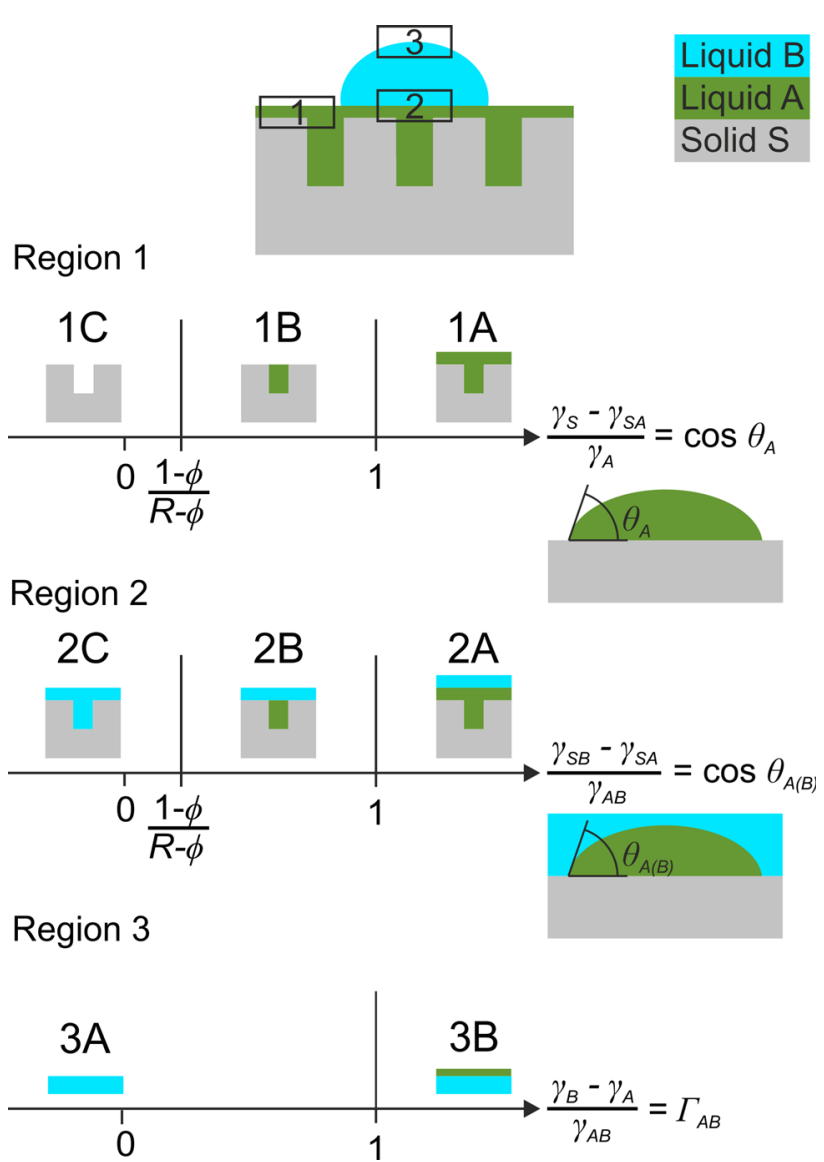

Fig. 3 Stability ranges of different wetting states $(A-C)$ in different regions (1-3) of a rough solid substrate $S$ infused by a liquid $A$ and wetted by an immiscible liquid $B$

(b) The probe liquid B should just float on top of liquid A and not displace liquid $\mathrm{A}$ and come in contact with the substrate at all (wetting state $2 \mathrm{~A}$ in Fig. 3). A pure liquid/liquid interface and a single liquid/liquid/air contact line guarantee minimal interaction and maximal mobility of liquid B on the surface.

(c) Liquids of low surface tension tend to engulf (cloak) drops of other liquids, resulting in the wetting state 3B in Fig. 3. Cloaking must be avoided, because it not only reduces the mobility of liquid $\mathrm{B}$ on the surface, but also removes liquid A from the substrate, eventually exposing the blank solid substrate to liquid B concomitant with a loss of its liquid-repellent properties.

Using the notation of Fig. 3, the desired wetting state of a liquid-repellent, liquid-infused substrate is therefore the state $(1 \mathrm{~A}, 2 \mathrm{~A}, 3 \mathrm{~A})$. The corresponding parameters are cos $\theta_{\mathrm{A}}=1 \quad\left(\theta_{\mathrm{A}}=0^{\circ}\right), \quad \cos \theta_{\mathrm{A}(\mathrm{B})}=1 \quad\left(\theta_{\mathrm{A}(\mathrm{B})}=0^{\circ}\right), \quad$ and $\Gamma_{\mathrm{AB}}<1$. We will discuss in the following if these requirements are practically achievable. Table 1 gives an overview of literature data on previously studied systems, 
Table 1 Wetting parameters $\cos \theta_{\mathrm{A}}, \cos \theta_{\mathrm{A}(\mathrm{B})}$, and $\Gamma_{\mathrm{AB}}$ of different liquid-infused substrates with a lubricating liquid A and a probe liquid B, calculated from published data of contact angles $\theta$, surface/interface tensions $\gamma$, and surface roughness $R$

\begin{tabular}{|c|c|c|c|c|c|c|}
\hline Solid & Liquid A & Liquid B & $\cos \theta_{\mathrm{A}}$ & $\cos \theta_{\mathrm{A}(\mathrm{B})}$ & $\Gamma_{\mathrm{AB}}$ & References \\
\hline Si-FDTS $^{\mathrm{a}}$ & Krytox & Hexadecane & 0.74 & 1.00 & 1.18 & {$[38]^{\mathrm{d}}$} \\
\hline Si-OTS $^{b}$ & Silicone & Water & 1.00 & 1.00 & 1.12 & [37] \\
\hline $\mathrm{Si}^{-O T S}{ }^{\mathrm{b}}$ & Krytox & Water & 0.88 & 0.88 & 1.12 & [39] \\
\hline Si-FDTS $^{\mathrm{a}}$ & Krytox & Glycerol & 0.74 & 1.00 & 1.06 & {$[38]^{\mathrm{d}}$} \\
\hline Si-FDTS $^{\mathrm{a}}$ & Krytox & Water & 0.74 & 0.89 & 0.98 & {$[38]^{\mathrm{d}}$} \\
\hline Si-FDTS $^{\mathrm{a}}$ & Krytox & Heptane & 0.74 & 0.79 & 0.66 & {$[38]^{\mathrm{d}}$} \\
\hline Si-OTS $^{b}$ & $\mathrm{Bmim}^{\mathrm{c}}$ & Water & 0.49 & 0.98 & 0.62 & {$[37]$} \\
\hline $\mathrm{Si}-\mathrm{SiO}_{2}$ & $\mathrm{Bmim}^{\mathrm{c}}$ & Water & 0.99 & -0.68 & 0.62 & [37] \\
\hline $\mathrm{Si}^{-\mathrm{SiO}_{2}}$ & Water & Hexane & 0.97 & 1.00 & -1.06 & {$[18]^{\mathrm{d}}$} \\
\hline
\end{tabular}

\footnotetext{
${ }^{a}$ Silicon coated with perfluorodecyltrichlorosilane

b Silicon coated with octadecyltrichlorosilane

c 1-Butyl-3-methylimidazolium bis(trifluoro-methyl-sulfonyl) imide

${ }^{\mathrm{d}} \cos \theta_{\mathrm{A}(\mathrm{B})}$ was calculated from the published $\Delta E_{1}$ values using the relationship $\Delta E_{1}=\gamma_{\mathrm{AB}}\left(R \cos \theta_{\mathrm{A}(\mathrm{B})}-1\right)$
}

where the parameters $\cos \theta_{\mathrm{A}}, \cos \theta_{\mathrm{A}(\mathrm{B})}$, and $\Gamma_{\mathrm{AB}}$ could be calculated from published data of contact angles and surface tensions. The systems are listed in Table 1 in the order of decreasing $\Gamma_{\mathrm{AB}}$ values. Apparently, the ideal wetting state $1 \mathrm{~A}, 2 \mathrm{~A}, 3 \mathrm{~A}$ is not reached in any of them. The upper half of them have $\Gamma_{\mathrm{AB}}$ values $\geq 1$ and suffer from the detrimental effects of cloaking (wetting state $3 \mathrm{~B}$ ). This includes many of the well-studied nonpolar SLIPS based on fluorinated substrate surfaces and polyfluorinated liquids like Krytox or FC70 as lubricating liquids [39]. The other groups in the lower part of Table 1 are polar SLIPS, which are characterized by a polar substrate like native silicon and a polar-infiltrating liquid like water. Only a few studies are known today about such polar liquid-infused systems, even though the parameters in Table 1 look promising: Especially the last example of native silicon infiltrated by water in contact with alkane solvents should come close to the ideal wetting state $(1 \mathrm{~A}, 2 \mathrm{~A}, 3 \mathrm{~A})$. A serious drawback, however, is the high volatility of water. A viable alternative should be a "water-like", hydrophilic ionic liquid with high surface tension. Based on these considerations, 1-ethyl-3-methylimidazolium methylsulfate $\left(\left[\mathrm{C}_{2} \mathrm{mim}\right] \mathrm{MeSO}_{4}\right)\left(\gamma=55 \mathrm{mN} \mathrm{m}^{-1}\right)$ was chosen for this study.

\section{Wetting properties of $\left[\mathrm{C}_{2} \mathrm{mim}\right] \mathrm{MeSO}_{4}$ on native and functionalized flat silicon}

To evaluate the suitability of different surface terminations of the silicon substrate for the infiltration with ionic liquid and the liquid-repellent properties of the resulting SLIPS for different probe liquids, the contact angles of $\left[\mathrm{C}_{2-}\right.$ mim] $\mathrm{MeSO}_{4}$ were measured on uncoated, flat silicon

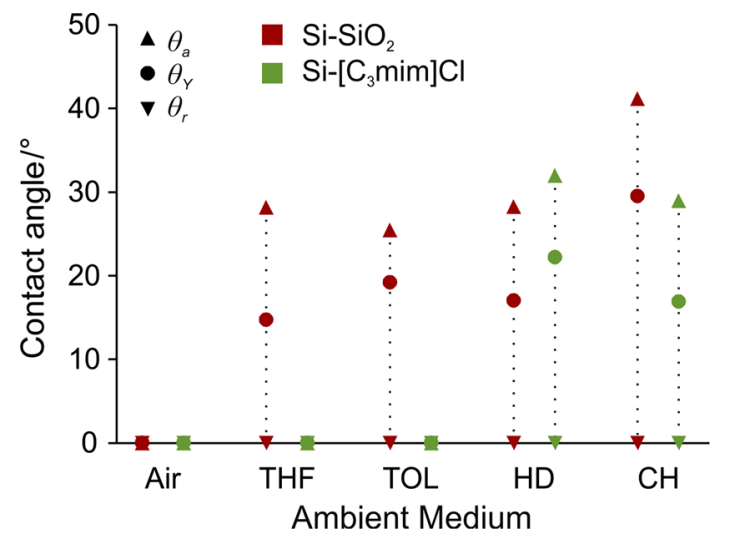

Fig. 4 Static contact angles $\left(\theta_{\mathrm{Y}}\right)$ and dynamic contact angles $\left(\theta_{\mathrm{a}}, \theta_{\mathrm{r}}\right)$ of the ionic liquid $\left[\mathrm{C}_{2} \mathrm{mim}\right] \mathrm{MeSO}_{4}$ on native silicon $\left(\mathrm{Si}-\mathrm{SiO}_{2}\right)$ and on functionalized silicon $\left(\mathrm{Si}-\left[\mathrm{C}_{3} \mathrm{mim}\right] \mathrm{Cl}\right)$ in different ambient media (THF tetrahydrofuran, TOL toluene, $\mathrm{HD}$ hexadecane, $\mathrm{CH}$ cyclohexane)

wafers covered with a native oxide layer $\left(\mathrm{Si}-\mathrm{SiO}_{2}\right)$ and on wafers coated with a monolayer of 1-methyl-3-(3trimethoxysilylpropyl)imidazolium chloride $\left(\mathrm{Si}-\left[\mathrm{C}_{3}-\right.\right.$ $\operatorname{mim}] \mathrm{Cl})$ in air and probe liquid as ambient medium (Fig. 4). In air as the ambient medium, $\left[\mathrm{C}_{2} \mathrm{mim}\right] \mathrm{MeSO}_{4}$ completely wets both the native silicon and the $\mathrm{Si}-\left[\mathrm{C}_{3-}\right.$ mim]Cl-functionalized flat silicon surface $\left(\theta_{\mathrm{Y}}=0^{\circ}\right)$. Thus, $\cos \theta_{\mathrm{A}}=1$ and a rough silicon substrate with either a native oxide or a $\mathrm{Si}-\left[\mathrm{C}_{3} \mathrm{mim}\right] \mathrm{Cl}$ surface layer should be completely wetted by $\left[\mathrm{C}_{2}\right.$ mim] $\mathrm{MeSO}_{4}$ (wetting state $1 \mathrm{~A}$ ). Stable SLIPS can therefore be prepared with both substrates. In a liquid environment, however, only the Si$\left[\mathrm{C}_{3} \mathrm{mim}\right] \mathrm{Cl}$ functionalized surface remains fully wetted by the ionic liquid in THF and toluene as ambient solvents $\left(\theta_{\mathrm{Y}}=0^{\circ}\right)$, resulting in $\cos \theta_{\mathrm{A}(\mathrm{B})}=1$ in these solvents and 
Table 2 Wetting parameters $\cos \theta_{\mathrm{A}}, \cos \theta_{\mathrm{A}(\mathrm{B})}$, and $\Gamma_{\mathrm{AB}}$ of nanoporous silicon infused with $\left[\mathrm{C}_{2} \mathrm{mim}\right] \mathrm{MeSO}_{4}$ (liquid A) with respect to different probe liquids (liquid B)

\begin{tabular}{|c|c|c|c|c|c|}
\hline Solid & Liquid A & Liquid B & $\cos \theta_{\mathrm{A}}$ & $\cos \theta_{\mathrm{A}(\mathrm{B})}$ & $\Gamma_{\mathrm{AB}}$ \\
\hline $\mathrm{Si}-\mathrm{SiO}_{2}$ & {$\left[\mathrm{C}_{2} \mathrm{mim}\right] \mathrm{MeSO}_{4}$} & Tetrahydrofuran & 1.00 & 0.97 & -4.4 \\
\hline $\mathrm{Si}-\mathrm{SiO}_{2}$ & {$\left[\mathrm{C}_{2} \mathrm{mim}\right] \mathrm{MeSO}_{4}$} & Toluene & 1.00 & 0.94 & -2.6 \\
\hline $\mathrm{Si}-\mathrm{SiO}_{2}$ & {$\left[\mathrm{C}_{2} \mathrm{mim}\right] \mathrm{MeSO}_{4}$} & Hexadecane & 1.00 & 0.86 & -1.3 \\
\hline $\mathrm{Si}-\mathrm{SiO}_{2}$ & {$\left[\mathrm{C}_{2} \mathrm{mim}\right] \mathrm{MeSO}_{4}$} & Cyclohexane & 1.00 & 0.97 & -1.6 \\
\hline $\mathrm{Si}\left[\mathrm{C}_{3} \mathrm{mim}\right] \mathrm{Cl}$ & {$\left[\mathrm{C}_{2} \mathrm{mim}\right] \mathrm{MeSO}_{4}$} & Tetrahydrofuran & 1.00 & 1.00 & -4.4 \\
\hline $\mathrm{Si}-\left[\mathrm{C}_{3} \operatorname{mim}\right] \mathrm{Cl}$ & {$\left[\mathrm{C}_{2} \mathrm{mim}\right] \mathrm{MeSO}_{4}$} & Toluene & 1.00 & 1.00 & -2.6 \\
\hline $\mathrm{Si}-\left[\mathrm{C}_{3} \mathrm{mim}\right] \mathrm{Cl}$ & {$\left[\mathrm{C}_{2} \mathrm{mim}\right] \mathrm{MeSO}_{4}$} & Hexadecane & 1.00 & 0.93 & -1.3 \\
\hline $\mathrm{Si}-\left[\mathrm{C}_{3} \mathrm{mim}\right] \mathrm{Cl}$ & {$\left[\mathrm{C}_{2} \mathrm{mim}\right] \mathrm{MeSO}_{4}$} & Cyclohexane & 1.00 & 0.96 & -1.6 \\
\hline
\end{tabular}

The solid surface composition was either a native oxide $\left(\mathrm{Si}-\mathrm{SiO}_{2}\right)$ or a monolayer of $\mathrm{Si}-\left[\mathrm{C}_{3} \mathrm{mim}\right] \mathrm{Cl}$

the desired wetting state $2 \mathrm{~A}$, where the probe liquid does not come in contact with the substrate at all. In all other systems included in Fig. 4, the static contact angles of $\left[\mathrm{C}_{2}\right.$ mim $] \mathrm{MeSO}_{4}$ lie between $10^{\circ}$ and $30^{\circ}$, such that $\cos$ $\theta_{\mathrm{A}(\mathrm{B})}<1$ and the probe liquid is predicted to partially replace the ionic liquid and come in direct contact with the solid substrate (wetting state 2B). A comparison of the IL contact angles in Fig. 4 between the uncoated, native substrate and the $\mathrm{Si}-\left[\mathrm{C}_{3} \mathrm{mim}\right] \mathrm{Cl}$-coated substrate reveals that the contact angles are generally lower for the coated substrates. Thus, an $\mathrm{Si}-\left[\mathrm{C}_{3} \mathrm{mim}\right] \mathrm{Cl}$ monolayer increases the "IL-philic" properties of the surface and favors in binary liquid systems the wetting by the ionic liquid. Table 2 lists the parameters $\cos \theta_{\mathrm{A}}, \cos \theta_{\mathrm{A}(\mathrm{B})}$, and $\Gamma_{\mathrm{AB}}$ for all systems under study here. The $\Gamma_{\mathrm{AB}}$ values were calculated from measured surface and interface tensions, which are listed in Table 3. A comparison with the literature data in Table 1 shows that the parameters in Table 2 are much closer to the desired configuration $\cos \theta_{\mathrm{A}}=1, \cos \theta_{\mathrm{A}(\mathrm{B})}=1, \Gamma_{\mathrm{AB}}<1$.

\section{Impregnation of nanoporous silicon with $\left[\mathrm{C}_{2} \mathrm{mim}\right] \mathrm{MeSO}_{4}$}

A few drops of $\left[\mathrm{C}_{2}\right.$ mim $] \mathrm{MeSO}_{4}$ were deposited on nanoporous silicon substrates with either native oxide or $\mathrm{Si}$ $\left[\mathrm{C}_{3} \mathrm{mim}\right] \mathrm{Cl}$ surface termination. In both cases, the ionic liquid is sucked into the substrate pores and the excess liquid spreads evenly across the surface, in agreement with the behavior predicted by the wetting parameter cos $\theta_{\mathrm{A}}=1$. Due to the extremely low volatility of the ionic liquid, a quasi-equilibrium state is reached when the excess liquid drains by gravity from the tilted substrate [27], leaving behind the impregnated, porous substrate with a liquid layer of a few micrometers thickness on top. Additionally, it was important here to saturate the infused ionic liquid with the particular probe liquid, because it has been shown that even minute quantities of dissolved cosolvents can change the surface tension and the wetting properties dramatically [39]. This was achieved here by immersing the substrate after IL impregnation vertically in the probe liquid. The whole process of coating and impregnation of the nanoporous substrates was monitored with FT-IR spectroscopy. Figure 5 shows IR spectra of the $\mathrm{Si}-\left[\mathrm{C}_{3}-\right.$ mim]Cl-coated substrates before impregnation (Fig. 5a) and after impregnation with $\left[\mathrm{C}_{2} \mathrm{mim}\right] \mathrm{MeSO}_{4}$ and equilibration in toluene (Fig. 5b). For comparison, spectra of the coating precursor $\left[\left(\mathrm{CH}_{3} \mathrm{O}\right)_{3} \mathrm{Si}-\left[\mathrm{C}_{3} \mathrm{mim}\right] \mathrm{Cl}\right.$ (Fig. $\left.5 \mathrm{~d}\right)$ and the neat ionic liquid $\left[\mathrm{C}_{2} \mathrm{mim}\right] \mathrm{MeSO}_{4}$ (Fig. 5c) are also included. The low-frequency region of these spectra is essentially identical, showing the highly characteristic absorption around $1577 \mathrm{~cm}^{-1}$ due to the $\mathrm{C}-\mathrm{C} / \mathrm{C}-\mathrm{N}$ ring stretching vibration of the imidazolium ring and the $\delta\left(\mathrm{CH}_{2}\right)$ deformation mode of the aliphatic hydrocarbon groups at about $1470 \mathrm{~cm}^{-1}[40,41]$. The $\mathrm{CH}$ stretching region shows absorptions of the aliphatic $\mathrm{CH}$ groups below $3000 \mathrm{~cm}^{-1}$ and the aromatic $\mathrm{CH}$ vibrations above $3000 \mathrm{~cm}^{-1}$ [41]. In addition, the $\mathrm{Si}-\left[\mathrm{C}_{3} \mathrm{mim}\right] \mathrm{Cl}$ monolayer spectrum shows a highly characteristic negative peak at $3747 \mathrm{~cm}^{-1}$, which indicates the removal of the free surface $\mathrm{OH}$ groups through covalent bond formation in the monolayer sample in comparison to the uncoated native silicon Ref. [42]. The disappearance of the free $\mathrm{OH}$ absorption in the monolayercoated samples also indicates a complete and homogeneous coverage of the outer and inner substrate surface with $\mathrm{Si}$ $\left[\mathrm{C}_{3} \mathrm{mim}\right] \mathrm{Cl}$. The $\mathrm{CH}$ stretching peaks in the monolayer spectrum are slightly different from the bulk reference spectrum (Fig. 5d) due to the overlap of additional methoxy group absorptions in the bulk spectrum. The ionic liquid spectra of the infused substrate saturated with toluene (Fig. 5b) and saturated with cyclohexane (not shown) are essentially identical to the reference spectrum of pure $\left[\mathrm{C}_{2} \mathrm{mim}\right] \mathrm{MeSO}_{4}$ (Fig. 5c) and show no absorptions of dissolved cosolvent. Hexadecane and tetrahydrofuran, on the other hand, show strong solvent absorptions in the saturated ionic liquid layer, which are due to the formation of an insoluble, slowly evaporating thin film of cosolvent at 


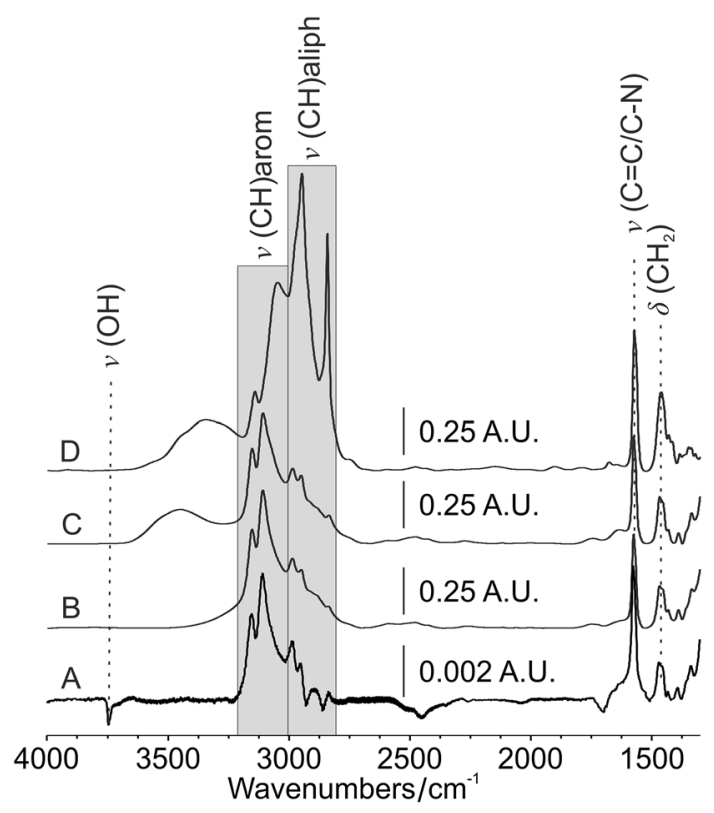

Fig. 5 IR spectra of $a$ nanoporous silicon coated with a monolayer of $\mathrm{Si}-\left[\mathrm{C}_{3} \mathrm{mim}\right] \mathrm{Cl}$ and $b$ infused with the ionic liquid $\left[\mathrm{C}_{2} \mathrm{mim}\right] \mathrm{MeSO}_{4}$. $c$ Reference spectrum of neat $\left[\mathrm{C}_{2} \mathrm{mim}\right] \mathrm{MeSO}_{4} . d$ Reference spectrum of neat $\left[\left(\mathrm{CH}_{3} \mathrm{O}\right)_{3} \mathrm{Si}-\left[\mathrm{C}_{3} \mathrm{mim}\right] \mathrm{Cl}\right.$. Spectra $a$ and $b$ were referenced against uncoated nanoporous silicon, spectra $c$ and $d$ against the empty beam

the ionic liquid/air interface, which is formed upon removal of the IL infused substrate from the IL/solvent mixture.

\section{Liquid repellency of $\left[\mathrm{C}_{2} \mathrm{mim}\right] \mathrm{MeSO}_{4}$-infused silicon}

Nanoporous silicon substrates infused with $\left[\mathrm{C}_{2-}\right.$ mim] $\mathrm{MeSO}_{4}$ and saturated with different probe liquids as described above were tested for their liquid repellency by depositing a drop of the probe liquid on the substrate surface and measuring first its static contact angle and then, by slowly tilting the substrate, the roll-off angle and the dynamic (advancing, receding) angles. Figure 6 shows images of a static and a moving drop (approximately, $20 \mathrm{~mm}^{3}$ ) of cyclohexane on the IL-infused substrate. Due to the high surface tension of $\left[\mathrm{C}_{2} \mathrm{mim}\right] \mathrm{MeSO}_{4}$ in comparison to cyclohexane, the static contact angle is very small $\left(\theta_{\mathrm{Y}}=6.4^{\circ} \pm 2.9^{\circ}\right)$ such that the drop is very flat and hardly visible in Fig. 6. Despite its large contact area with the IL-infused substrate, it is highly mobile and rolls off at a tilt angle of $\alpha=1^{\circ}$. Advancing and receding angles were measured as $\theta_{\mathrm{a}}=8.7^{\circ} \pm 4.0^{\circ}$ and $\theta_{\mathrm{r}}=4.0^{\circ} \pm 1.6^{\circ}$. Thus, $\Delta \theta \approx 4.7^{\circ}$ and $\alpha=1^{\circ}$, which are among the best liquidrepellent properties reported to date for low surface tension liquids. Very similar parameters were measured for toluene (Fig. $6 ; \Delta \theta \approx 10.6^{\circ}$ and $\alpha=2.5^{\circ}$ ). Hexadecane and tetrahydrofuran, on the other hand, do not form drops and wet the ionic liquid surface completely $\left(\theta_{\mathrm{Y}}=0^{\circ}\right)$. Notably, we did not observe any difference in the liquid-repellent properties between native silicon $\left(\mathrm{Si}-\mathrm{SiO}_{2}\right)$ and coated silicon substrates $\left(\mathrm{Si}-\left[\mathrm{C}_{3} \mathrm{mim}\right] \mathrm{Cl}\right)$, even though the cos $\theta_{\mathrm{A}(\mathrm{B})}$ parameters (Table 2 ) are different and predict partial replacement of the ionic liquid with both toluene and cyclohexane for the uncoated substrates. This should result in a noticeable increase of the roll-off angle $\alpha$ because of direct contact of the solvent with the solid surface. The fact that this was not observed in any of the systems investigated here suggests that the thermodynamic equilibrium state characterized by the static contact angles $\theta_{\mathrm{A}(\mathrm{B})}$ is not reached because of the kinetic barrier imposed by the receding angles. The ionic liquid can only be replaced by a solvent if the receding angle with the solvent as ambient medium $\theta_{\mathrm{r}, \mathrm{A}(\mathrm{B})}>0^{\circ}$. Since this is not the case in all systems studied here (Fig. 4), the ionic liquid never comes in contact with the solid and the ideal wetting state of a SLIPS substrate is maintained.

\section{Conclusions}

Liquid-infused, solid substrates (so-called SLIPS) are a novel and promising approach for the fabrication of stable, liquid-repellent surfaces, but are based almost exclusively on the use of fluorinated compounds as lubricating liquids and as surface-coating materials. We have shown in this study that hydrophilic, fluorine-free ionic liquids are a comparatively cheap and non-toxic alternative as lubricating liquids that can be used for the impregnation of natural, uncoated oxide surfaces. Due to the higher surface tensions of hydrophilic ionic liquids, another inherent problem of SLIPS with fluorinated lubricants-the cloaking of the probe liquid — can be avoided. On the other hand, the miscibility of hydrophilic ionic liquids with other solvents is generally higher, and the currently known examples of liquid-repellent polar SLIPS are limited to a relatively small group of immiscible solvents. Further studies focusing on a combined optimization of wetting and solubility are required here, making use of the large variety of physicochemical properties that ionic liquids offer.

\section{Experimental}

1-Methylimidazole (99\% purity) and (3-chloropropyl)trimethoxysilane ( $>97 \%$ purity) were purchased from Sigma-Aldrich and used as received. The solvents toluene (Aldrich, >99.3\%), hexadecane (Aldrich, 99\%), 


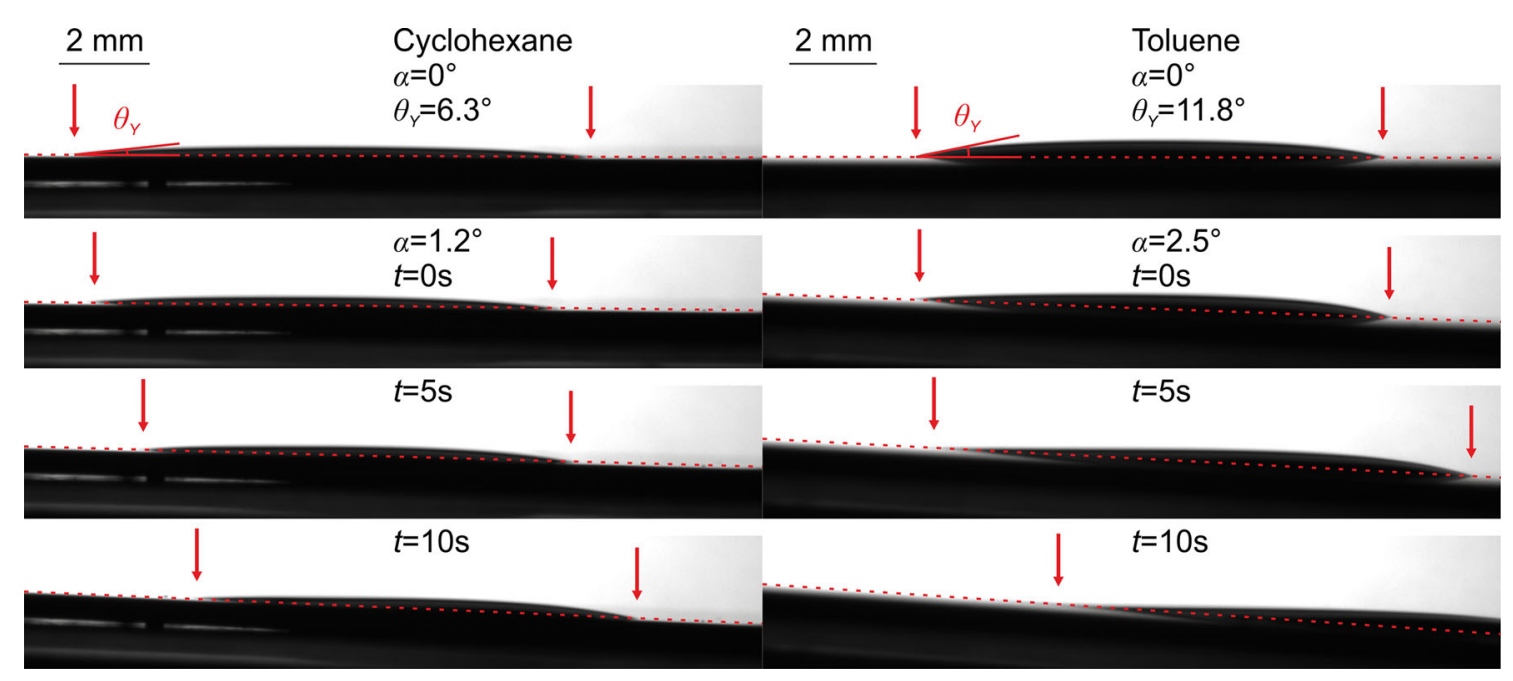

Fig. 6 Droplet images of cyclohexane and toluene on a nanoporous $\mathrm{Si}-\mathrm{SiO}_{2}$ substrate infused with $\left[\mathrm{C}_{2} \mathrm{mim}\right] \mathrm{MeSO}_{4}$. The upper row shows the static drops on the horizontal substrate with the Young

cyclohexane (Aldrich, >99\%), and tetrahydrofuran (Aldrich, $>99 \%$ ) were used as received.

Ionic liquids: 1-Methyl-3-(3-trimethoxysilylpropyl)imidazolium chloride $\left(\left[\left(\mathrm{CH}_{3} \mathrm{O}\right)_{3} \mathrm{Si}_{-} \mathrm{C}_{3} \mathrm{mim}\right] \mathrm{Cl}\right) \quad$ (1) was prepared according to a literature procedure [43]; analytical data were in accordance with literature [43]. 1-Ethyl-3methylimidazolium methylsulfate $\left(\left[\mathrm{C}_{2} \mathrm{mim}\right] \mathrm{MeSO}_{4}\right)$ (2) was prepared by adding $12.60 \mathrm{~g}$ methyl sulfate $(100 \mathrm{mmol})$ dropwise to a solution of $9.60 \mathrm{~g}$ freshly distilled 1-ethylimidazole $(100 \mathrm{mmol})$ in $100 \mathrm{~cm}^{3}$ dichloromethane under cooling at in an $\mathrm{NaCl} /$ ice bath. The solution was stirred at room temperature for $6 \mathrm{~h}$ and the solvent was removed under reduced pressure. The obtained liquid was dried in vacuo $\left(1 \times 10^{-2} \mathrm{mbar}\right)$ with stirring at $50{ }^{\circ} \mathrm{C}$ for $24 \mathrm{~h} ; 22.20 \mathrm{~g}(>99 \%)$ of a colorless liquid was yielded; analytical data were in accordance with literature [44].

Silicon wafers ((100)-oriented, p-doped, $10-20 \Omega \mathrm{cm}$, 500-550 $\mu \mathrm{m}$ thickness, double-sided polished) were purchased from MEMC Electronic Materials. They were cut into $18 \times 25 \mathrm{~mm}$ rectangular pieces and cleaned by sonication in toluene and UV-ozone treatment in a commercial cleaning chamber (Boekel Industries, UVClean). For the preparation of liquid-infused substrates, a nanoporous surface layer was created by silver-catalyzed electroless chemical etching [45] using aqueous $\mathrm{H}_{2} \mathrm{O}_{2} / \mathrm{HF}$ solutions as the etchant. The cleaned $\mathrm{Si}$ substrates were immersed in $20 \mathrm{~cm}^{3}$ aqueous solution of $17.0 \mathrm{mg}$ silver nitrate $\left(5 \mathrm{mmol} / \mathrm{dm}^{3}\right)$ and $4.5 \mathrm{~cm}^{3}$ hydrofluoric acid $(48 \%$, $6.2 \mathrm{mmol} / \mathrm{dm}^{3}$ ) for $1 \mathrm{~min}$ to deposit a layer of $\mathrm{Ag}$ nanoparticles to act as the catalyst in the etching process. After rinsing with water, the substrates were etched for angles $\theta_{\mathrm{Y}}$; the lower rows show a time sequence of the sliding drops beginning at the roll-off angle $\alpha$. Drop dimensions are marked by red arrows (color figure online)

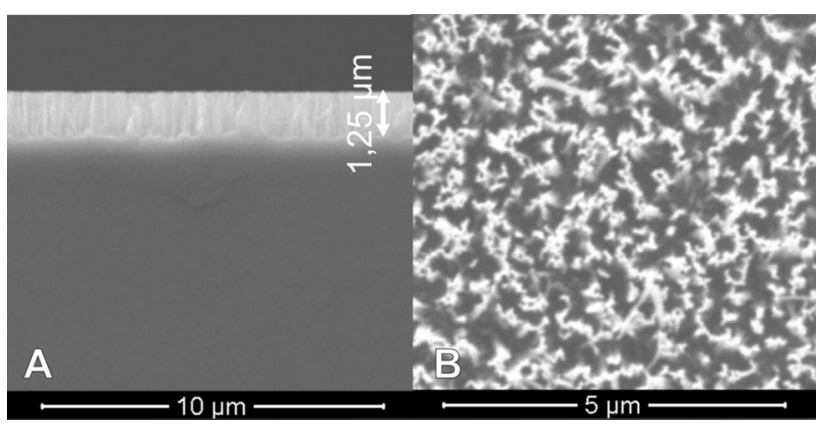

Fig. 7 Cross-sectional (a) and top view (b) SEM images of a nanoporous silicon substrate prepared by silver-catalyzed etching in $\mathrm{HF} / \mathrm{H}_{2} \mathrm{O}_{2}$ solution

$10 \mathrm{~min}$ in $20 \mathrm{~cm}^{3}$ of an aqueous solution of $4.5 \mathrm{~cm}^{3} \mathrm{HF}$ (48\%, $6.2 \mathrm{mmol} / \mathrm{dm}^{3}$ ) and $100 \mathrm{~mm}^{3}$ of $36 \% \mathrm{H}_{2} \mathrm{O}_{2}$ $\left(0.06 \mathrm{~mol} / \mathrm{dm}^{3}\right)$. The silver catalyst was finally dissolved by immersion in nitric acid (35\%) for $5 \mathrm{~min}$ and the samples were rinsed with water, immersed in piranha solution (4:1 $\left.\mathrm{H}_{2} \mathrm{SO}_{4} 96 \% / \mathrm{H}_{2} \mathrm{O}_{2} 36 \%\right)$, rinsed again with water and acetone and blow-dried with argon. This procedure yields a nanoporous surface layer of about $1 \mu \mathrm{m}$ thickness consisting of vertical, uniformly oriented nanowires of about $20-50 \mathrm{~nm}$ diameter separated by vertical pores of similar dimensions (Fig. 7).

\section{Surface modification}

The surface composition of both flat and nanoporous $\mathrm{Si}$ substrates in this study was either a surface oxide layer of about 1-2 nm thickness resulting from the UV-ozone 
treatment during the cleaning process or an adsorbed monolayer of 1-methyl-3-(3-trimethoxysilylpropyl)imidazolium chloride (1). The adsorption was carried out by immersing the cleaned substrates (flat or nanoporous) in a solution of $28.1 \mathrm{mg}\left(10^{-4} \mathrm{~mol}\right)$ of $\mathbf{1}$ in $10 \mathrm{~cm}^{3}$ toluene for 2 days. The samples were rinsed with toluene and immersed in $10 \mathrm{~cm}^{3}$ ethanol for 3 days to remove any physisorbed material. The samples were finally rinsed with ethanol and blow dried with argon. The thickness of the resulting films on flat silicon wafers were measured by ellipsometry and gave values of $4.4 \pm 0.6 \AA$, in agreement with the formation of a monolayer film. On nanoporous substrates, FT-IR was used to check the completeness of monolayer adsorption.

\section{Surface infiltration}

Freshly etched nanoporous silicon substrates were wetted on both sides with the ionic liquid $\left[\mathrm{C}_{2} \mathrm{mim}\right] \mathrm{MeSO}_{4}$ and the liquid was allowed to be sucked into the nanopores by capillary forces. To remove excess liquid and equilibrate the infused ionic liquid with a particular probe liquid (toluene, hexadecane, cyclohexane, tetrahydrofuran), the substrates were immersed vertically for 7 days in a twophase mixture of $1 \mathrm{~cm}^{3}$ of ionic liquid (covering just the very bottom of the immersed substrates) and $10 \mathrm{~cm}^{3}$ probe liquid. The samples were removed from the liquid mixture, the excess ionic liquid at the bottom of the substrate was carefully dabbed off and the substrates were immediately transferred into the sample chamber for contact angle measurements.

\section{NMR spectroscopy}

${ }^{1} \mathrm{H}$ NMR and ${ }^{13} \mathrm{C}$ NMR spectra were recorded on a Bruker AVANCE 250 spectrometer. The spectra were referenced internally to residual protio-solvent and solvent resonances, respectively, and were reported relative to tetramethylsilane $(\delta=0 \mathrm{ppm})$.

\section{IR spectroscopy}

Infrared spectra were measured on a Bruker Vertex 80 FTIR spectrometer using either a DTGS detector or a narrow band MCT detector. Between 16 and 256 scans at $4 \mathrm{~cm}^{-1}$ resolution were recorded for sample and reference, respectively. Dry and liquid-infused porous silicon substrates were measured in transmission at an incidence angle of $40^{\circ}$ to suppress residual interference fringes. Spectra of monolayer-coated porous silicon substrates were referenced against spectra of the uncoated substrate. Spectra of liquid reference compounds were measured as thin films between sodium chloride plates.

\section{Ellipsometry}

Ellipsometric measurements of coated and uncoated flat silicon samples were carried out using a Sentech SE 500adv single wavelength ellipsometer equipped with a $\mathrm{He}-\mathrm{Ne}$ laser and a rotating analyzer. The measured ellipsometric angles were converted into film thickness using the commercial instrument software based on the McCrackin algorithm. Optical constants of $n=3.864$ and $k=0.02$ for $\mathrm{Si}, n=1.465$ and $k=0$ for $\mathrm{SiO}_{2}$, and $n=1.50$ and $k=0$ for the organic coating layer were used. Measurements were carried out at four different spots across the sample surface and the variations were always below $1 \AA$.

\section{Scanning electron microscopy (SEM)}

SEM images were recorded on a FEI Quanta 200 MK2 electron microscope equipped with an Everhardt-Thornley detector. Working distances between 8 and $11 \mathrm{~mm}$ and an electron beam voltage of $10 \mathrm{keV}$ were used. For crosssectional measurements, the samples were freshly cleaved along a crystallographic axis with a diamond tip and were mounted with the cross section perpendicular to the electron beam.

\section{Contact angle and surface/interface tension measurements}

Contact angles and surface/interface tensions were measured on a Krüss DSA 30 contact angle goniometer equipped with a CCD video camera (resolution $780 \times 582$ pixel, speed $60 \mathrm{fps}$ ) and an inclinable sample stage with software-controlled tilting speed. A custom-built sample housing was used to carry out the contact angle and surface tension measurements in an atmosphere saturated with the probe liquid to minimize errors due to evaporation effects. Static contact angles were measured by putting a drop of probe liquid onto the sample surface, recording the drop image, and determining the contact angle using the tangent method $\left(\theta<10^{\circ}\right)$ or the Young-Laplace method $\left(\theta>10^{\circ}\right)$ of the instrument software. Advancing and receding angles were measured using the tilted plate method [46] by tilting the sample stage at a rate of $10^{\circ} / \mathrm{min}$ and continuously recording the image until the drop rolled off the surface. From the last image of the adhering drop, the advancing and receding angles were determined using the tangent method of the Krüss Advance software. Surface tensions were measured with the pendant drop method and a Young-Laplace drop shape analysis of the hanging drop in air as the surrounding medium. Interface tensions of the ionic liquid $\left[\mathrm{C}_{2} \mathrm{mim}\right] \mathrm{MeSO}_{4}$ in contact with different immiscible or partly miscible solvents were measured also 
Table 3 Surface tensions of different liquids and saturated liquid mixtures $(\mathrm{A}(\mathrm{B}) \rightarrow$ surface tension of liquid $\mathrm{A}$ saturated with B) and interface tensions of mutually saturated liquids

\begin{tabular}{|c|c|c|}
\hline Liquid & $\begin{array}{l}\text { Surface } \\
\text { tension } / 10^{-3} \mathrm{~N} \mathrm{~m}^{-1}\end{array}$ & $\begin{array}{l}\text { Interface } \\
\text { tension } / 10^{-3} \mathrm{~N} \mathrm{~m}^{-1}\end{array}$ \\
\hline$\left[\mathrm{C}_{2} \mathrm{mim}\right] \mathrm{MeSO}_{4}()$ & $55.2 \pm 0.5$ & \\
\hline$\left[\mathrm{C}_{2} \mathrm{mim}\right] \mathrm{MeSO}_{4}-\mathrm{TOL}$ & & $12.2 \pm 0.3$ \\
\hline TOL $\left(\left[\mathrm{C}_{2} \mathrm{mim}\right] \mathrm{MeSO}_{4}\right)$ & $25.0 \pm 0.1$ & \\
\hline$\left[\mathrm{C}_{2} \mathrm{mim}\right] \mathrm{MeSO}_{4}(\mathrm{TOL})$ & $56.6 \pm 0.4$ & \\
\hline$\left[\mathrm{C}_{2} \mathrm{mim}\right] \mathrm{MeSO}_{4}-\mathrm{HD}$ & & $20.5 \pm 0.4$ \\
\hline $\mathrm{HD}\left(\left[\mathrm{C}_{2} \mathrm{mim}\right] \mathrm{MeSO}_{4}\right)$ & $22.9 \pm 0.4$ & \\
\hline$\left[\mathrm{C}_{2} \mathrm{mim}\right] \mathrm{MeSO}_{4}(\mathrm{HD})$ & $51.1 \pm 0.6$ & \\
\hline$\left[\mathrm{C}_{2} \mathrm{mim}\right] \mathrm{MeSO}_{4}-\mathrm{CH}$ & & $18.2 \pm 0.4$ \\
\hline $\mathrm{CH}\left(\left[\mathrm{C}_{2} \mathrm{mim}\right] \mathrm{MeSO}_{4}\right)$ & $25.3 \pm 0.1$ & \\
\hline$\left[\mathrm{C}_{2} \mathrm{mim}\right] \mathrm{MeSO}_{4}(\mathrm{CH})$ & $53.4 \pm 0.7$ & \\
\hline$\left[\mathrm{C}_{2} \mathrm{mim}\right] \mathrm{MeSO}_{4}-\mathrm{THF}$ & & $7.4 \pm 0.2$ \\
\hline THF $\left(\left[\mathrm{C}_{2} \mathrm{mim}\right] \mathrm{MeSO}_{4}\right)$ & $23.3 \pm 0.2$ & \\
\hline$\left[\mathrm{C}_{2} \mathrm{mim}\right] \mathrm{MeSO}_{4}(\mathrm{THF})$ & $55.8 \pm 0.6$ & \\
\hline
\end{tabular}

Data represent mean values of three independent measurements by drop shape analysis of a pendant IL droplet submersed in the different solvents. Table 3 lists the surface tensions and interface tensions of all liquids and liquid mixtures employed in this study.

Acknowledgements Open access funding provided by TU Wien (TUW).

Open Access This article is distributed under the terms of the Creative Commons Attribution 4.0 International License (http:// creativecommons.org/licenses/by/4.0/), which permits unrestricted use, distribution, and reproduction in any medium, provided you give appropriate credit to the original author(s) and the source, provide a link to the Creative Commons license, and indicate if changes were made.

\section{References}

1. Young $\mathrm{T}$ (1805) Philos Trans R Soc Lond 95:65

2. Gao L, McCarthy TJ (2006) Langmuir 22:6234

3. Extrand CW (2003) Langmuir 19:3793

4. Chu Z, Seeger S (2014) Chem Soc Rev 43:2784

5. Liu M, Zheng Y, Zhai J, Jiang L (2010) Acc Chem Res 43:368

6. Bellanger H, Darmanin T, Taffin E, Guittard F (2014) Chem Rev 114:2694

7. Kota AK, Mabry JM, Tuteja A (2013) Surf Innov 1:71

8. Cassie ABD, Baxter S (1944) Trans Faraday Soc 40:54

9. Nosonovsky M, Bhushan B (2009) Philos Trans R Soc 367:1511

10. Tuteja A, Choi W, Ma M, Mabry JM, Mazzella SA, Rutledge GC, McKinley GH, Cohen RE (2007) Science 318:1618

11. Wenzel RN (1936) Ind Eng Chem 28:988

12. Pan S, Kota AK, Mabry JM, Tuteja A (2013) J Am Chem Soc 135:578

13. Lee SE, Kim HJ, Lee SH, Choi DG (2013) Langmuir 29:8070

14. Golovin K, Lee DH, Mabry JM, Tuteja A (2013) Angew Chem Int Ed 52:13007

15. Deng X, Mammen L, Butt HJ, Vollmer D (2012) Science 335:67

16. Zhang JP, Seeger S (2011) Angew Chem Int Ed 50:6652
17. Liu T, Kim C (2014) Science $346: 1096$

18. Wong TS, Kang SH, Tang SKY, Smythe EJ, Hatton BD, Grinthal A, Aizenberg J (2011) Nature 477:443

19. Glavan AC, Martinez RV, Subramaniam AB, Yoon HJ, Nunes RMD, Lange H, Thuo MM, Whitesides GM (2013) Adv Funct Mater 24:60

20. Daniel D, Mankin MN, Belisle RA, Wong TS, Aizenberg J (2013) Appl Phys Lett 102:231603

21. Vogel N, Belisle RA, Hatton B, Wong TS, Aizenberg J (2013) Nat Comm 4:2176

22. Epstein AK, Wong TS, Belisle RA, Boggs EM, Aizenberg J (2012) Proc Natl Acad Sci 109:13182

23. Grinthal A, Aizenberg J (2014) Chem Mater 26:698

24. Hou X, Hu Y, Grinthal A, Khan M, Aizenberg J (2015) Nature 519:70

25. Li J, Kleintschek T, Rieder A, Cheng Y, Baumbach T, Obst U, Schwartz T, Levkin PA (2013) ACS Appl Mater Interfaces $5: 6704$

26. MacCallum N, Howell C, Kim P, Sun D, Friedlander R, Ranisau J, Ahanotu O, Lin JJ, Vena A, Hatton B, Wong TS, Aizenberg J (2015) ACS Biomater Sci Eng 1:43

27. Lafuma A, Quere D (2011) Eur Phys Lett 96:56001

28. Zhang J, Wang A, Seeger S (2014) Adv Funct Mater 24:1074

29. Stone HA (2012) ACS Nano 6:6536

30. Rykaczewski K, Anand S, Subramanyam SB, Varanasi KK (2013) Langmuir 29:5230

31. Kim P, Wong TS, Alvarenga J, Kreder MJ, Adorno-Martinez WE, Aizenberg J (2012) ACS Nano 6:6569

32. Han H, Huang Z, Lee W (2014) Nano Today 9:271

33. Peng K, Fang H, Hu J, Wu Y, Zhu J, Yan Y, Lee S (2006) Chem Eur J 12:7942

34. Henriksson A, Friedbacher G, Hoffmann H (2011) Langmuir 27:7345

35. Kolbeck C, Lehmann J, Lovelock KRJ, Cremer T, Paape N, Wasserscheid P, Fröba AP, Maier F, Steinrück HP (2010) J Phys Chem B 114:17025

36. Tariq M, Freire MG, Saramago B, Coutinho JAP, Lopes JNC, Rebelo LPN (2012) Chem Soc Rev 41:829

37. Smith JD, Dhiman R, Anand S, Reza-Garduno E, Cohen RE, McKinley GH, Varanasi KK (2013) Soft Matter 9:1772

38. Dai X, Stogin BB, Yang S, Wong TS (2015) ACS Nano 9:2015 
39. Anand S, Paxson AT, Dhiman R, Smith JD, Varanasi KK (2012) ACS Nano 6:10122

40. Sobota M, Nikiforidis I, Hieringer W, Paape N, Happel M, Steinrück HP, Görling A, Wasserscheid P, Laurin M, Libuda J (2010) Langmuir 26:7199

41. Höfft O, Bahr S, Kempter V (2008) Langmuir 24:11562

42. Tripp CP, Hair ML (1991) Langmuir 7:923
43. Zheng X, Luo S, Zhang L, Cheng JP (2009) Green Chem 11:455

44. Holbrey JD, Rechert WM, Swatloski R, Broker GA, Pitner WR, Seddon KR, Rogers RD (2002) Green Chem 4:407

45. Zhang ML, Peng KQ, Fan X, Jie JS, Zhang RQ, Lee ST, Wong NB (2008) J Phys Chem C 112:4444

46. Extrand CW, Kumagai Y (1997) J Colloid Interface Sci 191:378 